\title{
An Experimental Study of Various Network Protocols and Understanding its Implementation in Real World
}

\author{
Prof. Romi Morzelona \\ Professor, Department of Computer Science, \\ Russia
}

Article Info

Page Number: $122-127$

Publication Issue:

Vol 71 No. 1 (2022)

Article History

Article Received: 18 November 2021

Revised: 03 December 2021

Accepted: 19 December 2021

Publication: 28 January 2022

\begin{abstract}
This paper will explain the medium and set of rules through which data is exchanged during communication in the network. The set of rules which are followed during the implementation of the network for the data communication is called as network protocol. For any network to work efficiently there is need to define certain set of rules and standards which should be followed. There are many layers in a network and each layer will have specific protocol that if followed for the exchange of the data. This paper will explain the types of protocols available, structure of data message, and also the challenges occurring in implementation of the protocols.
\end{abstract}

Keywords: - Network protocol, types of protocol, Data Message, Challenges.

Introduction: - The set of rules and standards defined is called as protocol. In a network, for data exchange we need medium through which data will be transferred which is commonly known as network. The process by which it is determined that how that information or data will be exchanged is called as network protocol. In a network, there is one sender who will send messages to the other end of the network which is called as receiver of the data. The protocol will define certain rules by which the message should be transferred between the sender and the receiver in the network. Before the exchange of data takes place, it is decided by both the ends, which rules to be followed. This results in smooth transaction of the data.

Why do we need protocols? -

The protocols are set of defines rules and regulations which makes the data exchange easy and identifies how it will be transferred. The functioning of protocols can be understood by its layer. Each layer has certain function so the rules will be unique to each layer. Following are three levels of protocols: - 


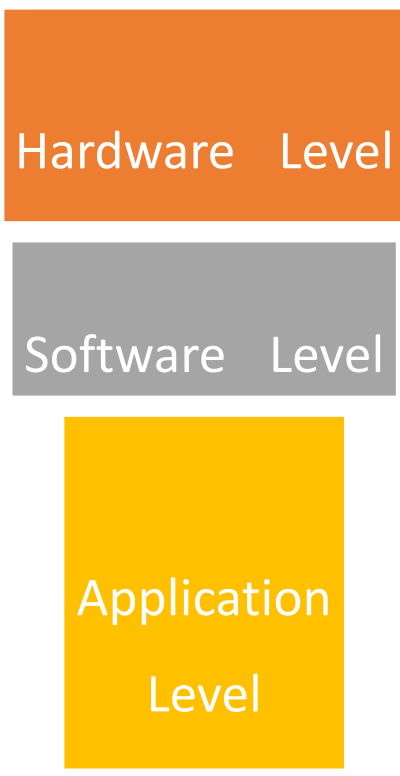

Figure 1 Types of level of protocols.

1. Hardware Level: - At this level, the protocol will define set of rules that how the hardware devices connected to each other in a network will exchange data with each other. So, protocol in this level will be implemented at the hardware level of the system.

2. Software Level: - Certain set of rules and standards will be implemented for the flow of information between software of the system. Protocols at this level will facilitate the flow of data between the software of the system.

3. Application Level: -At this level, the protocols allow various applications of the system to work to serve their purpose.

Components of Network protocols: - [2]

The components of the protocols are related to the data message structure. It defines the syntax and structure of the data in which it will be transmitted using protocols. Following are the three main elements of the protocols: -

a. Syntax: - The syntax of the data message will define the format and structure of the data message with which it is transmitted. It defines how to manipulate the data which is exchanged.

b. Semantics: - This element is related to the manipulation or interpretation of the data. The data will be sent in the form of fields which consists of bits and bytes. Semantic will determine the meaning of each bit and byte of the field in order to understand the patterns and to decide what step and action should be taken on the basis of those bits and bytes.

c. Time Constraint: - It is also one of the key elements of the data exchange between the sender and the receiver. It is related to the timing of the data being send. It will help to understand the time at which the message is sent and also the speed and accuracy of the data by which it is being sent.

Characteristics of Protocols: -

$>$ No medium required: - The protocol is connection less and does not require any medium to implement it as these are the rules and specifications by which the data should be exchanged between the sender and the receiver. 
Data dependant - The type of protocol used to exchange the data depends upon the type of the data being transferred.

Reliable exchange of data: - If all the set of rules and regulations of the protocol is followed properly then there is less chance of the loss of the data. The network protocols are the source of proper transmission of the data.

Types of Protocols: -

The network protocols depend upon the type of data being exchanged and the level on which it is exchanged. There are three types of network protocols which are explained below: - [3]

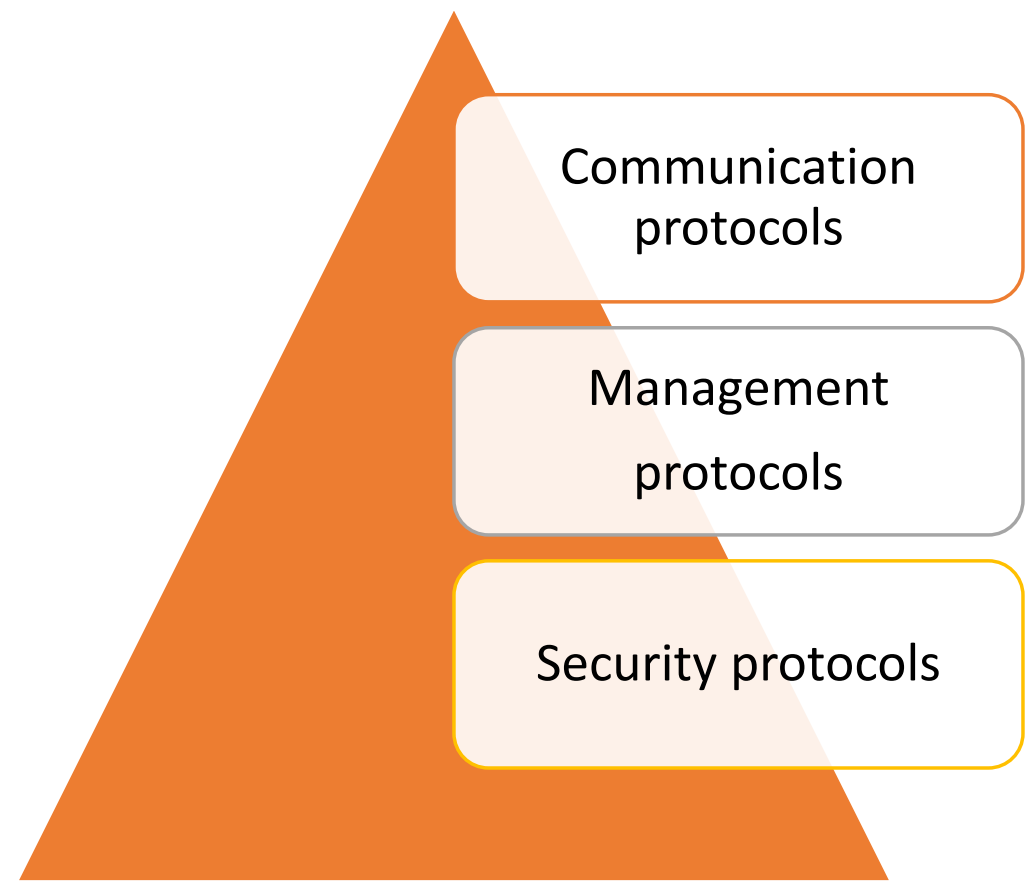

Figure2 Categories of Network protcols.

1. Communication protocols: - These protocols identify about how the information will be transferred between the receiver and the sender in the network. If these communication protocols do not set the rules of the protocols, then the data being exchanged will not be communicated between the two ends of the network. As we know, that there are two types of communication which are analogy and digital, so the responsibility of the communication protocols will be to check if there are any errors, they need to detect the authorisation of the data being exchanged and also make sure that the syntax, semantic of the data message is done properly. Following are the protocol examples of communication protocols:

a. TCP:-Transmission Control Protocol: - This type of protocol is responsible for the transmission of the data between the sender and receiver of the network. The role is to divide the data into small data packets and then sent across the network for the receiver to receive it. The working of the TCP protocol is a two-way communication. First of all, the data is divided into small data packets. Then the client will send its packet over the network to other side of the network. The data packet will have IP address of the server. When the server receives the packet then it will send acknowledgement message by sending a message to the client. And then the client will also send the reply by sending its own acknowledgement message. 
b. UDP: - User datagram protocol also helps to transfer the data between two devices in the network. It also works similar to that of TCP protocol. But the difference between the two is that the connection is made in TCP protocol by sending messages and then the actual communication will take place but in UDP prior connection will not be made.

c. HTTP: - This is hyper-text transmission protocol which works at the application layer of the network and is responsible for the reliable exchange of data like media, files, documents over web. The client will send request messages to the server to load or respond with particular web page and the related server with respond with the web page being loaded.

d. IRC: - Internet Relay Chat: -It is used to exchange the text messages over the internet. This method of protocols can be used by the network which will have many distributed devices among which the information is being exchanged.

2. Management protocols: -This category of the protocols helps in managing the performance of the network. It defines set of policies and methods which are used to monitor and maintain the network. It is a continuous approach which helps to determine the availability of the resources in the network. It also helps to determine error created during the exchange of the messages. It also identifies any data loss, connectivity issues. Basically, it deals with the management of the data being exchanged in the network. Following are the examples of the management protocols: -

a. SNMP: -Simple network managing protocol: - This protocol is used to manage the monitoring of the network devices which are present in the network. It is also used to identify any error messages or data lost during the information sharing. It can also be used to configure the device remotely. Components of SNMP protocols are: - [4]

$>$ SNMP manager: -It is manager which is centralised and used to manage and monitor the network devices.

SNMP agent: - It is a software management which is used to manage the network devices like routers, switches etc.

MIS: - It is management information base which consists of the essential details of the network devices which it is supposed to manage.

b. ICMP: - Internet control Management protocol is the set of rules and standards which is used to identify the errors created during the exchange of the information and also to report them. It will detect the error $r$ connection loss and will help to send the messages which will indicate the error or data loss.

3. Network Security Protocols: -The role of this protocol is to make sure that the data exchanged over the internet is safe and secure and there are no security attacks. These protocols will use various methods of encryption and cryptography to make sure that the data is secure. It will also make sure that there is no unauthorised access to the information being shared. The task of this protocol is to identify whether the access is authorised or unauthorised. In case of unauthorised access, it will reject and discard that particular data message. Following are the types of security protocols: -

$>$ SSL: - Secure socket protocol is the protocol which will use encrypted messages in order to save them and keep them safe and non - readable. It primary function is to make sure that the connections are safe to transfer the data. 
$>$ SFTP: - Secure file transfer protocol is to make sure that the filles transmitted over the network are safe and secure. This will first use the authentication messages to check the authentication of the client and the server and once it is confirmed then only the files will be shared among the client and the server. It will discard the message if any one of the clients or the server is found to be unauthorised.

$>$ HTTPS: - Secure hypertext transfer protocol: - The role of this protocol is to make sure that the data like images, files, etc shared on the web is safe and secure. It is to make sure that the web page being loaded is from secured and safe server. This will also use encryption methods to increase the security level of the data.

Functions of Protocols: - [5]

$>$ Connectivity: - The role of the protocol is to make sure that the network is either connectionless or connected. If the amount of data being exchanges is more then it is the responsibility of the protocol to implement connected network for the reliable transfer of the data and the data will not be lost in connected network.

$>$ Data Packets: - In a network for the reliable transfer of the data, the protocols will segment the data into small data packages with header which will consists of the IP address of the receiver. And on the receivers end it will be reassembled. So, the function of the protocol is to segment and reassemble the data packets.

$>$ Error Control: - It is the role of the protocol to ensure that the data transferred is correct and not giving errors. The protocols also make sure that if there are any error messages then it should have the capability to identify them and then discard them so that wrong messages should not be sent.

$>$ Time issue: - It is the responsibility of the protocols to make sure that the data transferred does not take longer time for its transmission. The transfer of the data should be smooth and on time for increasing the efficiency of the network.

Data flow: - It is related to the transmission of the data to its correct receiver. It is the duty of the protocol that the data is received by the dedicated receiver.

Conclusion: - It is observed that for any network to work efficiently there should be certain rules which should be followed. These are called protocols. There are varieties of protocols which performs unique function. The type of protocol being used depends on the type of the network in which the data is being transferred. The role of protocol is to facilitate the transfer of data and make sure that it is transferred safely. It is also used to manage and monitor the network for any data loss, connection failures etc. Hence it is very important to choose the right protocol for the network in an organisation.

\section{References: -}

[1].https://afteracademy.com/blog/what-are-protocols-and-what-are-the-key-elements-of-protocols

[2].https://practice.geeksforgeeks.org/problems/what-are-the-key-elements-of-protocols

[3].https://www.cdw.com/content/cdw/en/articles/networking/types-of-network-protocols.html

[4].https://www.geeksforgeeks.org/simple-network-management-protocol-snmp/

[5].https://www.tutorialspoint.com/what-is-the-function-of-protocol 
[6].Ronad, A. ., and M. . Madgi. "Online Platform for Agricultural Produce Livestock Marketing". International Journal on Recent and Innovation Trends in Computing and Communication, vol. 9, no. 12, Dec. 2021, pp. 12-18, doi:10.17762/ijritcc.v9i12.5494.

[7]. Omara , I. . (2022). Gamification and Its Application in Social Network Implementation. International Journal of New Practices in Management and Engineering, 10(02), 01-04. https://doi.org/10.17762/ijnpme.v10i02.130 